\title{
Seroprevalence of Cystic Echinococcosis and related risk factors for infection among children in a rural community in Fars Province, Southern Iran
}

\author{
Bahador Sarkari $^{\mathrm{a}, \mathrm{b}, *}$, Nasir Arefkhah ${ }^{\mathrm{a}}$, Fariba Ghorbani ${ }^{\mathrm{a}}$, Farzaneh Meskini ${ }^{\mathrm{a}}$, Narjes Yektaeian ${ }^{\mathrm{a}}$, \\ Sepehr Shahriarirad ${ }^{\mathrm{c}}$, Zohreh Mostafavi-Pour ${ }^{\mathrm{d}}$ \\ ${ }^{a}$ Department of Parasitology and Mycology, School of Medicine, Shiraz University of Medical Sciences, Shiraz, Iran \\ ${ }^{\mathrm{b}}$ Basic Sciences in Infectious Diseases Research Center, Shiraz University of Medical Sciences, Shiraz, Iran \\ ${ }^{\mathrm{c}}$ Student Research Committee, School of Medicine, Shiraz University of Medical Sciences, Shiraz, Iran \\ ${ }^{\mathrm{d}}$ Recombinant Proteins Laboratory, Department of Biochemistry, School of Medicine, Shiraz University of Medical Sciences, Shiraz, Iran
}

\section{A R T I C L E I N F O}

\section{Keywords:}

Hydatid cyst

Children

Seroprevalence

Fars province

Iran

\begin{abstract}
A B S T R A C T
Background: Cystic Echinococcosis (CE) or hydatid cyst is one of the most common diseases between humans and livestock that is caused by the larval stage of Echinococcus granulosus. The disease is a major health and also economic concern in Iran. Most of the studies on CE in different areas of Iran have been conducted in adults and there is little information about the prevalence of the disease in children.

Methods: This cross-sectional study was conducted to find out the seroprevalence of hydatid cyst in children in a rural community in Fars province, southern Iran. Children sera samples were evaluated for anti-hydatid cyst antibodies; using a recombinant B8/1 antigen of $E$. graunlosus in an ELISA system.

Subjects: Blood samples were taken from 578 children living in three villages in Sarmashhad district in Fars province, southern Iran.

Results: Of the 578 recruited children, 298 (51.6\%) were boys and 280 (48.4\%) were girls. The mean age of the children studied was 6.8 ( \pm 3.7 ) years. Anti-hydatid cyst antibodies were detected in sera of 39 out of 578 children, corresponding to a seroprevalence rate of $6.7 \%$. While age, educational level, keeping dogs in the household, and residential areas had no significant influence on the risk of CE infection (p $>0.05$ ), the correlation between sex and seropositivity to CE was significant ( $\mathrm{p}<0.05)$.

Conclusion: Findings of this study showed that the rate of seroprevalence of hydatid cyst in children in the rural community is high. The study suggests that preventive measurements including the education of the population should begin in early childhood as a large percentage of the adult CE patients are resulted from childhood infection.
\end{abstract}

\section{Introduction}

Cystic Echinococcosis (CE) or hydatid cyst is one of the most important diseases between humans and animals, which is caused by the larval stage Echinococcus granulosus. ${ }^{1}$ The mature adult worm lives in the small intestine of dogs and other carnivores, as the final host, while sheep, cattle, goats, and humans serve as its intermediate hosts. Humans become infected through ingestion of food, vegetation, and water contaminated with the parasite's eggs. ${ }^{2}$

$\mathrm{CE}$ has a worldwide distribution and is reported from all tropical, temperate, and subtropical countries. ${ }^{3}$ In Iran, the disease has been reported throughout the country, while its prevalence is lower in the arid and southern regions. The seroprevalence of CE has been reported from $1.6 \%$ to more than $20 \%$ in various provinces of Iran. ${ }^{4-9}$ The yearly number of surgeries owing to CE in Iran has been reported to be 1295 cases, with an average annual surgical incidence of $0.61-2.6$ per 100,000 populations in different geographical regions of the country. ${ }^{10}$

In Iran, various studies have been conducted regarding the seroprevalence and also the surgical cases of hydatid cyst. ${ }^{11-13}$ The pooled estimate of the seroprevalence of CE in Iran has been estimated at $6.0 \%$ (9). The seroprevalence rates are reported to be $3.4 \%$ in Alborz Province, in central, $2.6 \%$ in Lorestan province in western, $1.79 \%$ in Meshkinshahr in Northwest, and 7.2\% in Ysuj district in the southwest of the country. ${ }^{14-16}$

Nevertheless, most of the studies on hydatid cyst in different areas of Iran have been conducted in adults' population, and therefore

\footnotetext{
* Corresponding author. Department of Parasitology and Mycology, School of Medicine, Shiraz University of Medical Sciences, Shiraz, Iran.

E-mail address: sarkarib@sums.ac.ir (B. Sarkari).
} 
information on the seroprevalence of this disease in children is very limited. Since people in the Iranian rural communities living on animal husbandry and they usually keep dogs in their households, the conditions are favorable for the development of the Echinococcus life cycle and the risk of transmission of the disease is high, especially in the low age groups of the community. Considering the aforementioned points, the current study aimed to assess the seroprevalence of CE among the children in a rural community in Fars province in southern Iran.

\section{Methods}

\subsection{Study area and sampling}

The study was carried out in 2017 in three rural communities, Sar Mashhad, ToleSaman and HosseinAbad, of Kazeroon Township in Fars province, southern Iran. People in these three rural areas are mainly living on agriculture and animal husbandry. While stray dogs are roaming around, dog maintenance is also common in the households of the area. Dog-associated zoonotic diseases are common in the area. ${ }^{17-20}$ After receiving approval from the Ethical Review Committee of Shiraz University of Medical Sciences (ethical code: IR.SUMS.Med.REC.1396. S322), and following obtaining the informed consent from the children parents, blood samples (about $5 \mathrm{~mL}$ ) were collected from 578 children in three rural communities in the area. From about 800 children living in those three villages, 578 participated in the study and these were all of the children who showed up after general calling by health care workers in the area. Blood samples were taken from children who showed up in the health center in each village. Sera were separated from the blood and transferred to the serological laboratory at Department of Parasitology in Shiraz University of Medical Sciences and stored at $-20^{\circ} \mathrm{C}$ until tested. The enrollment criteria were being the resident of the studied area, while the exclusion criteria were having a fever, normally not feeling healthy, and reluctant to provide the blood. During sampling, a questionnaire was used to obtain the data regarding the children gender, age, residence, educational level and risk factors linked to the hydatid cyst.

\subsection{Detection of anti-hydatid cyst antibodies}

Sera samples were evaluated for anti-hydatid cyst antibodies in an ELISA system, using a recombinant B8/1 antigen of E. granulosus. ${ }^{21}$ The $\mathrm{B} 8 / 1$ recombinant antigen has been produced in our previous study which had a sensitivity of $93 \%$ and specificity of $92 \%$ for the diagnosis of human CE. To perform the ELISA with the recombinant antigen, flatbottom 96 -well microplates were coated $(100 \mu \mathrm{l} /$ well) with $5 \mu \mathrm{g} / \mathrm{mL}$ of the recombinant antigen in coating buffer $(0.1 \mathrm{M}$ carbonate/bicarbonate). Unbound antigens were removed by washing buffer (phosphate buffered saline, containing 0.05\% Tween 20; PBST) and blocking was performed, using 3\% skimmed milk. Serum samples (1:100 dilutions in PBST) were added to the plates and the plates were washed after $2 \mathrm{~h}$. Horseradish peroxidase-conjugated anti-human antibody (Sigma, USA) (100 $\mu \mathrm{L}$ at 1:4000 dilution) was added to the plates and were then washed after $1.5 \mathrm{~h}$. A substrate $(100 \mu \mathrm{L} /$ well of $0.4 \mathrm{mg} / \mathrm{mL}$ OPD, $0.3 \%$ $\mathrm{H}_{2} \mathrm{O}_{2}$ in $0.1 \mathrm{M}$ citrate buffer, $\mathrm{pH} 5$ ) was added to the plate and the absorbance (at $490 \mathrm{~nm}$ ) was measured, using a microplate reader (BioTek, ELx800). Positive sera (from pathologically confirmed hydatid cyst patients) along with sera from healthy control subjects were applied in each run of ELISA and the cutoff point was set at 2 standard deviations (SD) above the mean OD of the negative control samples.

\subsection{Statistical analyses}

Statistical analysis of the data was performed using SPSS (Ver. 20). The association between the seropositivity to hydatid cyst and children demographic features and also the risk factors for the infection were verified, utilizing the chi-square test.
Table 1

Demographic features and relative seropositivity to hydatid cyst in children in rural communities in Fars Province, Southern Iran.

\begin{tabular}{|c|c|c|c|c|c|}
\hline \multirow[t]{2}{*}{ Characteristics } & \multirow[t]{2}{*}{$\begin{array}{l}\text { Frequency } \\
\text { (No.) }\end{array}$} & \multirow[t]{2}{*}{ Percent (\%) } & \multicolumn{2}{|c|}{$\begin{array}{l}\text { Positive for anti- } \\
\text { hydatid cyst antibodies }\end{array}$} & \multirow[t]{2}{*}{$P$ value } \\
\hline & & & $\begin{array}{l}\text { Frequency } \\
\text { (No.) }\end{array}$ & $\begin{array}{l}\text { Percent } \\
(\%)\end{array}$ & \\
\hline \multicolumn{6}{|l|}{ Gender } \\
\hline Male & 298 & 51.6 & 14 & 4.7 & 0.031 \\
\hline Female & 280 & 48.4 & 25 & 8.9 & \\
\hline \multicolumn{6}{|l|}{ Age group } \\
\hline$\leq 5$ & 230 & 39.8 & 12 & 5.2 & 0.535 \\
\hline $6-10$ & 224 & 38.8 & 16 & 7.1 & \\
\hline $11-15$ & 121 & 20.9 & 11 & 9.1 & \\
\hline $16-20$ & 3 & 0.5 & 0 & 0 & \\
\hline \multicolumn{6}{|l|}{ Residence } \\
\hline Sar Mashhad & 471 & 77.0 & 33 & 7 & 0.603 \\
\hline ToleSaman & 94 & 15.1 & 6 & 6.4 & \\
\hline HosseinAbad & 13 & 7.9 & 0 & 0 & \\
\hline \multicolumn{6}{|l|}{ Educational levels } \\
\hline Non-School aging & 234 & 40.5 & 12 & 5.1 & 0.438 \\
\hline Elementary level & 330 & 57.1 & 26 & 7.9 & \\
\hline Secondary level & 14 & 2.4 & 1 & 7.1 & \\
\hline \multicolumn{6}{|c|}{ Presence of dogs in the household } \\
\hline Yes & 344 & 59.5 & 26 & 7.6 & 0.221 \\
\hline No & 234 & 40.5 & 13 & 5.6 & \\
\hline
\end{tabular}

\section{Results}

Of the 578 recruited children, 298 (51.6\%) were boys and 280 (48.4\%) were girls. The mean age of the children studied was 6.8 ( \pm 3.7 ) years. Most of the subjects $(39.8 \%)$ were in the age group equal to or less than 5 years old and the majority of the subjects (57.1\%) were in their primary educational level. Anti-hydatid cyst antibodies were detected in the sera of 39 out of 578 children, corresponding to a seroprevalence rate of $6.7 \%$. While age, educational level, keeping dogs, and residential areas had no significant influence on the risk of hydatid cyst infection $(p>0.05)$, the correlation between sex and seropositivity to hydatid cyst was statistically significant ( $\mathrm{p}<0.05)$.

Seroprevalence to hydatid cyst was more prevalent in the age group of 11-15 years $(9.1 \%)$, however, there were no associations between seropositivity to hydatid cyst and age of the participants ( $\mathrm{p}>0.05$ ). Table 1 shows the demographic features and relative seropositivity to hydatid cyst in children in rural communities in Fars Province, Southern Iran.

\section{Discussion}

Hydatid cyst is an important public health problem in children, in terms of its prevalence and also of its disease characteristics. The disease in children has important epidemiological significances associated with severity, transmission, and also prevention interventions. It has been shown that in areas where the prevalence of hydatid cyst in adults is high, this prevalence is also high in children. Hydatid cyst is mostly acquired in childhood, but the disease is more common during adolescence. It has been reported that, contrary to adults, the incidence of pulmonary hydatid cyst is much frequent in children and combined lung and liver involvement is more common in children than adults. ${ }^{22}$ Aslanabadi et al. reviewed the hospital records of 59 children with hydatidosis referring to Tabriz children hospital, Tabriz, Iran and found that lung hydatidosis is more common than hepatic hydatidosis in children. ${ }^{23}$

In a retrospective analysis of 31 children with $\mathrm{CE}$ in Tehran, Iran, lungs involvement was much common (77\%) than liver. ${ }^{24}$ It has also been shown that the surgical complications of CE are less frequent and the disease prognosis is satisfactory in children. ${ }^{22}$ Furthermore, comparison of pulmonary hydatid disease between children and adults showed that giant pulmonary hydatid cysts are more commonly found 
in children. ${ }^{25} \mathrm{~A}$ study in Bulgaria showed that hydatid cyst in children aged 0-9 years can be seen most frequently in the lungs, in contrast to patients from other age groups. ${ }^{26}$

In our study, seropositivity to hydatid cyst in children in the studied region was $6.7 \%$. This rate of infection is higher than those reported in some of the other areas of Iran. ${ }^{8,27}$ The main reason for this difference is that the current study was conducted in children living in a rural area while most of the previous studies have been conducted on adults and mainly in urban regions. Moreover, the relatively high rate of seropositivity to hydatid cyst in the asymptomatic children in the current study might be attributed to the presence of an enormous population of stray dogs in the region. Stray dogs roaming around in areas like playgrounds which are preferred places for children to play, as a result, they are continuously exposed to the Echinococcus eggs.

In our study, seropositivity to hydatid cyst was higher in females $(8.9 \%)$ than in males $(4.7 \%)$. Such observation has been reported in some of the previous studies. ${ }^{7,28,29}$ For instance, a retrospective analysis of hospital records of children with hydatid cyst in the south of Iran showed that the frequencies of hydatid cyst in females (56.1\%) are higher than males (42.1\%). ${ }^{30}$ Contrary to our findings, studies by Mamishi et al. and also Aslanabadi et al. in Iran and Kandeel et al. in Egypt reported a higher prevalence of hydatid cyst in males. ${ }^{23,24,31}$

In our study, seropositivity to CE showed an overall increasing trend with age, although the association between age and seropositivity to CE was not statistically significant. The trend of increasing of seropositivity rate and age has also been reported in other studies. ${ }^{32}$

ELISA, using native antigen $\mathrm{B}(\mathrm{AgB})$, is the most common method for seroepidemiological studies of hydatid cyst. ${ }^{7,14,16,21,27,33}$ In the current study, we applied a recombinant B8/1 subunit of antigen B which has a higher diagnostic value, as compared with the native $\mathrm{AgB}$, for the detection of hydatid specific antibodies. The recombinant antigen has a sensitivity of $92 \%$ and a specificity of $93 \%$ in the diagnosis of human hydatid cyst. ${ }^{35}$ Considering that the efficiency of recombinant antigen in detecting of hydatid cyst antibodies is more than that of the native counterpart, the positive cases reported in this study are more reliable.

In hydatid cyst, antibody titer may remain for years, even after surgical removal of the cyst or proper drug treatment. Therefore a positive serological test may not necessarily imply the presence of an active cyst or the reactivation of CE. Differentiation of past (cured, calcified or asymptomatic cyst) from the present (active or progressive) hydatid infection is difficult by the existing antibody detection assays. ${ }^{34}$ Using the imaging approaches in seropositive cases with sign or symptoms would confirm the active form of the disease.

It is also worth mentioning that the seropositivity to hydatid cyst may or may not be linked to the further development of hydatid disease, since a primary exposure and seroconversion may lead to progressive or abortive cysts or spontaneous cure. Therefore, the seropositive children of this study may never show an acute or progressive form of the disease.

\section{Conclusion}

Findings of the current study revealed a relatively high rate of seropositivity to hydatid cyst in the region and indicate that $\mathrm{CE}$ is a significant health problem for children, especially in rural communities. The study suggests that preventive measurements including the education of the population should begin in early school-age children, as most of the hydatid cyst infections acquire during childhood. Moreover, the portable ultrasound examination of the children may confirm the presence of hydatid cyst in the seropositive cases.

\section{Conflicts of interest}

None.

\section{Acknowledgments}

The authors would like to thank the children and also their parents who collaborated on blood sample donations. The study was funded by the vice chancellor for research and technology at Shiraz University of Medical Sciences, Iran (Grant No. 97-01-01-17401). The study was the subject of the medical dissertation of Dr. Farzaneh Meskini. The technical assistance of Mr. Salarikia is acknowledged.

\section{References}

1. Romig T, Deplazes P, Jenkins D, et al. Ecology and life cycle patterns of Echinococcus species. Adv Parasitol. 2017;95:213-314.

2. Craig PS, Hegglin D, Lightowlers MW, Torgerson PR, Wang Q. Echinococcosis: control and prevention. Adv Parasitol. 2017;96:55-158.

3. Deplazes P, Rinaldi L, Alvarez Rojas CA, et al. Global distribution of alveolar and cystic echinococcosis. Adv Parasitol. 2017;95:315-493.

4. Galeh TM, Spotin A, Mahami-Oskouei M, et al. The seroprevalence rate and population genetic structure of human cystic echinococcosis in the Middle East: a systematic review and meta-analysis. Int J Surg. 2018;51:39-48.

5. Rafiei A, Hemadi A, Maraghi S, Kaikhaei B, Craig P. Human cystic echinococcosis in nomads of the south-west Islamic Republic of Iran. East Mediterr Health J. 2007;13:41-48.

6. Sarkari B, Ghobakhloo N, Moshfea A, Eilami O. Seroprevalence of human fasciolosis in a new-emerging focus of fasciolosis in Yasuj district, southwest of Iran. Iran J Parasitol. 2012;7(2):15-20.

7. Sarkari B, Hosseini F, Khabisi SA, Sedaghat F. Seroprevalence of cystic echinococcosis in blood donors in Fars province, Southern Iran. Parasite Epidemiol Control. 2016;15(1):8-12 2.

8. Sarkari B, Sadjjadi SM, Beheshtian MM, Aghaee M, Sedaghat F. Human cystic echinococcosis in Yasuj District in Southwest of Iran: an epidemiological study of seroprevalence and surgical cases over a ten-year period. Zoonoses Public Health 2010;57(2):146-150.

9. Shafiei R, Teshnizi SH. The seroprevalence of human cystic echinococcosis in Iran: a systematic review and meta-analysis study. J Parasitol Res. 2016;2016:1425147.

10. Fasihi Harandi M, Budke CM, Rostami S. The monetary burden of cystic echinococcosis in Iran. PLoS Neglected Trop Dis. 2012;6(11):e1915.

11. Ebrahimipour M, Budke CM, Najjari M, Yaghoobi K. Surgically managed human cystic echinococcosis in north-eastern Iran: a single center's experience from 2001 to 2008. J Parasit Dis. 2017;41(3):883-887.

12. Harandi MF, Moazezi SS, Saba M, et al. Sonographical and serological survey of human cystic echinococcosis and analysis of risk factors associated with seroconversion in rural communities of Kerman, Iran. Zoonoses Public Health. 2011;58(8):582-588

13. Sarkari B, Fatemie Sfedan A, Moshfe A, et al. Clinical and molecular evaluation of a case of giant primary splenic hydatid cyst: a case report. Iran J Parasitol. 2016;11(4):585-590.

14. Dabaghzadeh H, Bairami A, Kia EB, Aryaeipour M, Rokni MB. Seroprevalence of human cystic echinococcosis in Alborz Province, central Iran in 2015. Iran J Public Health. 2018;47(4):561-566.

15. Fallah Omrani V, Rouhani S, Kazemi B, Seyyedtabaei SJ, Kheirandish F, Rezapour M. Seroprevalence of IgG antibodies against Echinococcus granulosus by ELISA method using recombinant Agb in Lorestan province, western Iran. Iran J Public Health. 2017;46(8):1132-1138.

16. Kalantar K, Gholami M, Mirzaee G, et al. Seroepidemiological study of human hydatidosis in Meshkinshahr district, Ardabil province, Iran. J Parasitol Res. 2011;6(3):19-25.

17. Sarkari B, Hatam G, Ghatee M. Epidemiological features of visceral leishmaniasis in Fars province, southern Iran. Iran J Public Health. 2012;41(4):94.

18. Sarkari B, Naraki T, Ghatee MA, Abdolahi Khabisi S, Davami MH. Visceral leishmaniasis in Southwestern Iran: a retrospective clinico-hematological analysis of 380 consecutive hospitalized cases (1999-2014). PLoS One. 2016;11(3):e0150406.

19. Gigloo AL, Sarkari B, Rezaei Z, Hassan M. Asymptomatic Leishmania infected children: a seroprevalence and molecular survey in a rural area of Fars Province, Southern Iran. J Trop Med. 2018;2018:1-6.

20. Sarkari B, Alirezaei R, Layegh Gigloo A, et al. Seroprevalence and risk factors for Toxocara infection among children in a rural community in Fars province, southern Iran. Parasite Immunol. 2018:e12582.

21. Savardashtaki A, Sarkari B, Arianfar F, Mostafavi-Pour Z. Immunodiagnostic value of Echinococcus granulosus recombinant B8/1 subunit of antigen B. Iranian J Immunol. 2017;14(2):111-122.

22. Talaiezadeh AH, Maraghi S. Hydatid disease in children: a different pattern than adults. Pakistan J Med Sci. 2006;22(3):329

23. Aslanabadi S, Zarrintan S, Abdoli-Oskouei S, et al. Hydatid cyst in children: a 10-year experience from Iran. Afr J Paediatr Surg. 2013;10(2):140-144.

24. Mamishi S, Sagheb S, Pourakbari B. Hydatid disease in Iranian children. J Microbiol Immunol Infect. 2007;40(5):428-431.

25. Montazeri V, Sokouti M, Rashidi MR. Comparison of pulmonary hydatid disease between children and adults. Tanaffos. 2007;6(1):13-18.

26. Jordanova DP, Harizanov RN, Kaftandjiev IT, Rainova IG, Kantardjiev TV. Cystic echinococcosis in Bulgaria 1996-2013, with emphasis on childhood infections. Eur J Clin Microbiol Infect Dis. 2015;34(7):1423-1428. 
27. Rahimi H, Sadjjadi S, Sarkari B. Performance of antigen B isolated from different hosts and cyst locations in diagnosis of cystic echinococcosis. Iran J Parasitol. 2011;6(1):12-19.

28. Cai H, Guan Y, Ma X, et al. Epidemiology of echinococcosis among schoolchildren in Golog Tibetan autonomous prefecture, Qinghai, China. Am J Trop Med Hyg. 2017;96(3):674-679.

29. Vlad DC, Neghina AM, Dumitrascu V, Marincu I, Neghina R, Calma CL. Cystic echinococcosis in children and adults: a seven-year comparative study in western Romania. Foodb Pathog Dis. 2013;10(2):189-195.

30. Sanaei Dashti A, Kadivar MR, Alborzi A, et al. Analysis of hospital records of children with hydatid cyst in south of Iran. J Parasit Dis. 2017;41(4):1044-1048.

31. Kandeel A, Ahmed ES, Helmy H, El Setouhy M, Craig PS, Ramzy RM. A retrospective hospital study of human cystic echinococcosis in Egypt. East Mediterr Health J. 2004;10(3):349-357.

32. Fomda BA, Khan A, Thokar MA, et al. Sero-epidemiological survey of human cystic echinococcosis in Kashmir, North India. PLoS One. 2015;10(4):e0124813.

33. Saberi-Firouzi M, Kaffashian F, Hayati E, et al. Prevalence of hydatidosis in nomadic tribes of southern Iran. Med J Islam Repub Iran. 1998;12(2):113-118.

34. Sarkari B, Rezaei Z. Immunodiagnosis of human hydatid disease: where do we stand? World J Methodol. 2015;5(4):185-195. 